\title{
The impact of food industry reformulation, innovation and consumer preference on dietary intakes in Ireland: a probabilistic intake model
}

\section{Abstract}

Strong evidence exists linking poor diet to increased risk of overweight, obesity and non-communicable diseases. Reformulation initiatives, whereby the food industry gradually reduces energy, sodium, sugar, fat and saturated fat content of their products, are identified as important strategies to improve dietary intakes. The analysis aimed to examine the impact of voluntary reformulation, changes to products on the market and changes in consumer preferences on dietary intakes in Ireland from 2005 to 2017. Data on composition and volume sales $(\mathrm{kg} /$ year) of products available on the market in 2005 and 2017 were collected from 15 Food Drink Ireland (FDI) member companies via online templates. These products were assigned to appropriate food and beverage groups identified in four Irish University Nutrition Alliance (IUNA) surveys of preschool children (1-4 years), children (5-12 years), teenagers (13-17 years) and adults (18-90 years). Assignment of FDI products to IUNA foods and beverages was carried out using weighted distributions for a given group of foods. The weightings were taken from the sales volumes of similar products relative to one another in a given category in a given year. Monte Carlo simulations were used to run the IUNA survey consumption data with both sets of weighted composition data from 2005 and 2017. The Creme Global intake model was used to estimate daily energy and nutrient intakes for all four populations during 2005 and 2017. The Wilcoxon-signed rank test was used to test for differences between the two years. Changes in both the products available on the market and market share of these products were observed from 2005 to 2017. The nutrient with the greatest intake reduction between the two years for all ages was sugar. Children and teens were the most affected, where total sugar intakes reduced by $3.2 \mathrm{~g} / \mathrm{d}$ and $2.7 \mathrm{~g} / \mathrm{d}$, respectively. This reduction was primarily driven by the beverage category. There were modest saturated fat intake reductions observed for teens and adults $(0.2 \mathrm{~g} / \mathrm{d}$ and $0.5 \mathrm{~g} / \mathrm{d}$, respectively). Energy, total fat and sodium intakes for all ages remained relatively stable between the two years. This analysis highlights the impact of not only food industry efforts but also consumer choices on nutrient intakes in Ireland. It is worth noting that the data collected predates the sugar tax on sugar-sweetened beverages in Ireland. Reductions in sugar intakes were not compensated by total fat or energy increases.

\section{Conflict of Interest}

There is no conflict of interest 